\title{
The Extreme Energy Events observatory: status and perspectives
}

Ivan Gnesi* on behalf of the EEE Collaboration

"E. Fermi" Center, Rome and Phys. Dep. and INFN Turin, Italy

E-mail: gnesi@to.infn.it

Extreme Energy Events is a inhomogeneus array of high time resolution tracking telescopes extending over 10 degrees in latitude and longitude. Because of its unique and unconventional features the experiment is searching for rare and exotics cosmic ray-related phenomena, like Extensive Air Showers Long Distance Correlations, as well as addressing middle and low energy topics, like local anisotropies, sun activities survey and life-related issues. The observatory has also a strong outreach impacts, being the telescopes installed in High Schools buildings, where teachers and students contribute to a real extended cosmic ray experiment. An overview of the observatory status is given and first results on the various topics are discussed.

35th International Cosmic Ray Conference - ICRC2017

10-20 July, 2017

Bexco, Busan, Korea

${ }^{*}$ Speaker. 


\section{Introduction}

The Extreme Energy Events (EEE) Project [1,2] is aimed to the study of Cosmic Rays (CR) and Cosmic Rays-related phenomena, via a synchronous sparse array of 53 tracing detectors, deployed over a broad area covering more than 10 degrees in latitude and 11 in longitude. The observatory is being upgraded regularly: 10 stations are being installed within the end of the year, leading to a $20 \%$ acceptance increase. In figure 1 the present displacement of the EEE stations is shown. 47 stations out of 53 are installed inside High School buildings, where teachers and students contribute to the experiment activities while also addressing Modern Physics by participating to a real CR observatory [3].

The EEE array is composed by both clusters and stand-alone stations, each made of three Multigap Resistive Plate Chambers (MRPC), a CR dedicated version of the module used as the Time Of Flight (TOF) detector at the ALICE experiment $[4,5]$. The result is a sparse array where each detection site is made of few detectors at $30 \mathrm{~m}-2 \mathrm{~km}$ distance from the others. Currently the largest distance between the EEE telescopes is about $1200 \mathrm{~km}$ (between CERN laboratories and Catania). Data collected by the single stations are sent to the CNAF center, the biggest computing facility of the italian National Institute of Nuclear Physics (INFN), where they are stored, reconstructed and made available for being analysed. At present the experiment has closed the "RUN 3", in June 2017; the whole data set collected since fall 2014 has already exceeded 48 billions of muon tracks.

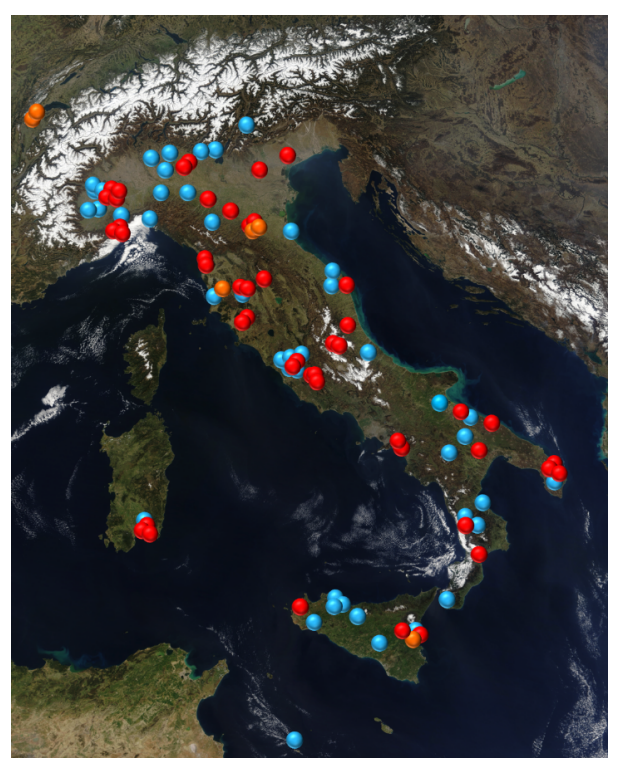

Figure 1: Distribution of the 53 EEE stations. Red points: stations installed inside High School buildings. Orange point: stations installed in research laboratories (CERN and INFN sections). Blue points: High Schools in waiting list for a station.

\section{The EEE technology and data treatment}

The EEE stations and their performances have been already described in [6, 7]. Here, we give 
a brief description of both chambers technology, data transmission, validation and reconstruction.

\subsection{The telescopes}

Each EEE telescope is made of three Multigap Resistive Plate Chambers (MRPCs), mounted on a frame at a typical distance of $0.5 \mathrm{~m}$. The final acceptance of a telescope is $\frac{\pi}{2} \mathrm{srad}$ for the downward flying particles and $\pi$ srad if we consider upward flux, that can be measured and identified by the TOF measurement. An MRPCs consists of six gas gaps, as shown in figure 2a. The structure consists of two thicker glass plates (1.9 mm thick), coated with resistive paint, and five thinner glass plates ( $1.1 \mathrm{~mm}$ thick), spaced by $300 \mu \mathrm{m}$ by means of commercial nylon fishing line; the typical glass resistivity is $\sim 10^{13} \Omega \mathrm{cm}$. The MRPC readout is obtained by applying 24 copper readout strips, $2.5 \mathrm{~cm}$-wide and $3.2 \mathrm{~cm}$ pitch, on the top and bottom fiberglass panels. The active area is finally $0.82 \times 1.58 \mathrm{~m}^{2}$. Each chamber is flushed with $98 \% \mathrm{C}_{2} \mathrm{H}_{2} \mathrm{~F}_{4}$ plus $2 \% \mathrm{SF}_{6}$ gas mixture. The chambers are operated in avalanche mode with a typical operating voltage around 18 $\mathrm{kV}$ supplied by DC/DC converters. The measured MRPC efficiency is typically $95 \%$. The signal induced on the copper strips is processed by a fast discriminator-amplifier Front-End card (FEA) [8] at the two ends of the chamber. A six-fold coincidence of the signals from the left and right sides of the three MRPC of the telescope generates the data acquisition trigger. Particle hits are firstly determined by the strips involved and by the time difference of signal arrival at the right and left FEA, respectively. The time is measured by two multi-hit TDCs (CAEN Mod. V1190A/B), operated at $100 \mathrm{ps}$ bin width, providing an overall spatial resolution of about $1 \mathrm{~cm}$ along the two coordinates. Beside the local hit time the absolute time of each event is recorded and synchronized by means of Global Positioning System (GPS) modules, in order to get the event time stamp and allowing multi-telescope analysis. A picture of a EEE telescope is shown in figure $2 b$.

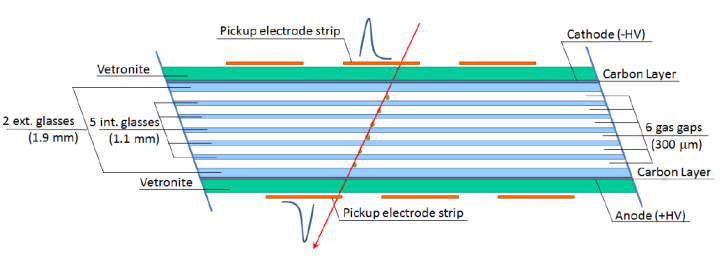

(a)

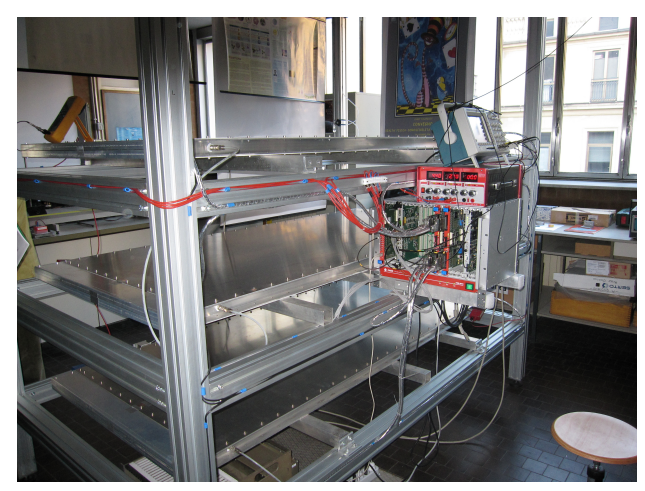

(b)

Figure 2: a). The internal structures of a MRPC plane (see description in the text). b). The TORI04 telescopes. The three MRPC planes are shown. The VME crate on the right houses the TDC modules, the trigger card and the USB bridge card for the communication to the DAQ PC. 


\subsection{Reconstruction and Data validation}

The data-processing infrastructure for the EEE experiment is provided by CNAF (Centro Nazionale Analisi Fotogrammi), the central computer facility of the Italian National Institute for Nuclear Physics (INFN) and one of most prominent centers for distributed computing in Italy. The data acquisition is organized in units (or runs) of 50,000 events each automatically transferred to CNAF and processed by virtual machines mounted on demand out of a cloud-based system. The event reconstruction is automatically performed and provides data summary tape (DST) with tracks direction and absolute time information, available for both calibration and scientific analysis purposes. The reconstruction pipeline runs in parallel a Data Quality Monitor (DQM) system, providing summary reports for each telescope with both hardware survey and data validation purposes.

\section{Results overview}

The EEE main fields of investigation extend over a broad energy range and topics. From the study of local muon flux dependance on solar activity to the investigation of the upward-going component of muon looking for rare signals; from the search for anisotropies at various scales to the observation of EAS. Finally, thanks to the broad and non-uniform distribution of EEE telescope clusters, the search for possible long distance EAS correlations (LDC) can be addressed.

\subsection{Galactic Cosmic Ray flux Decreases (Forbush effect)}

The monitoring of galactic cosmic ray flux decreases is of interest for understanding phenomena occurring on the solar heliosphere, as well as on other observable stars. As it is known, they are related to the emission of mass from star corona and often to solar flares, even if such relation is not completely understood, especially the interplay with interplanetary structures [10,11]. The effect on the solar wind directly affects the measured galactic cosmic ray flux on Earth, giving typical flux fluctuations of a few percent on a few days basis.

The long term survey of cosmic ray flux fluctuations has been historically performed by neutron monitors (NM), showing usually higher angular acceptance and lower energy threshold than EEE muon tracking detectors. On the other side the overall acceptance of each EEE station, the timing resolution better than $30 \mathrm{~ns}$ and the tracking capabilities allow for the study of the muon component of the GCRDs, opening to comparison with NM and to a deeper understanding of such phenomena. Four variations have been already observed by the EEE telescopes in 2011, 2012, 2014 and 2015, by correcting the absolute flux for the known sources of systematic uncertainties, mainly due to pressure fluctuations above the telescopes. The results show that the EEE array has the capability of becoming a stable survey for GCRDs over a broad latitude and longitude range. In Figure 3 a GCRD occurred in November 2014 and observed by 6 EEE stations is shown in comparison with the OULU NM.

\subsection{Local Anisotropies}

Galactic cosmic rays are expected to be nearly isotropic, due to their interaction with the galactic magnetic field over the long path to the Earth. Observation of cosmic rays at energies smaller than $10^{15} \mathrm{eV}$ is a useful tool to inspect the magnetic field structure in the interstellar medium 


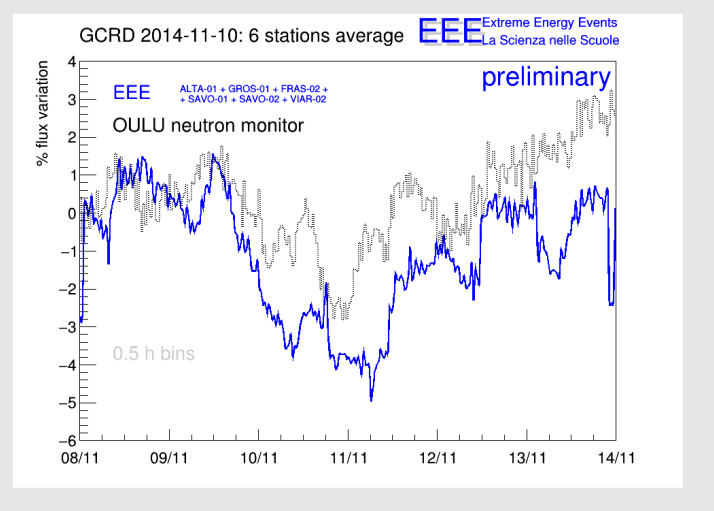

Figure 3: Blue: GCRD observed by 6 EEE teelscopes. Black: OULU Neutron Monitor data.

of our Solar System. Small anisotropies may be induced by large scale as well as local magnetic field features. At $1 \mathrm{TeV}$, sizeable effects from the eliosphere may be expected on the cosmic ray distributions. The so-called Compton-Getting effect, introduced in 1935 [12], predicts a dipole component in the cosmic ray anisotropy, due to the relative motion of the observer with the isotropic cosmic ray plasma rest frame: i.e. the anisotropy due to Earth motion around the Sun has been observed by several experiments to be of the order of $\sim 10^{-4}$.

A first analysis with a dataset collected by the EEE network has been performed, providing a skymap in equatorial coordinates, for the dominating sub-TeV extensive air showers. More than $100 \mathrm{M}$ events were fully reconstructed after track quality selection. Raw data were corrected for the time exposure of each telescope and its geometrical acceptance by the scrambling method, and corrected maps were extracted and plotted in the Right Ascension vs Declination coordinates. In figure 4 the comprehensive sky map for the four telescopes involved in the analysis is shown. Corrected data maps are compatible with isotropic distributions at the level of $5 \times 10^{-3}-10^{-2}$, being compatible with statistical fluctuations associated to the number of analysed events. The available statistics is now 2 order of magnitudes larger than the one used for obtaining these results, allowing for inhomogeneities down to $10^{-3}$. Additional analyses are being performed to search for possible deviations from an isotropic pattern at a higher confidence level.

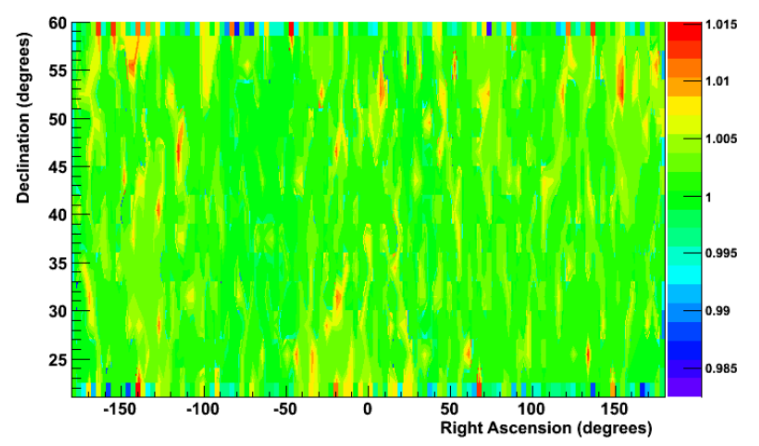

Figure 4: Corrected skymap with the whole data set from the telescopes CAGL-01, CATA-01, SAVO-01, TRIN-01. 


\subsection{First results on EAS}

The search and identification of EAS is fundamental for exotic effects to be detected by the EEE Observatory. The main feature of the EEE telescopes is related to the tracking-timing capabilities, which are under study for the implementation of the primary cosmic ray carachterization. The telescopes are displaced in clusters, made of two or more telescopes, and single stations, which are tenths of kilometers far from the other stations.

The study of EAS has been preliminarly addressed by studying the time-coincidence rate between two telescopes in the same cluster as a function of the distance. In Figure 5a the two-telescopes rate vs distance is shown. The coincidences between two events in different telescopes of the same cluster is obtained by also applying a directional cut (10-20 depending on the relative distance) between the 2 tracks. The time difference is also corrected by the path difference due to the arrival direction of the shower disk w.r.t. the vertical. At energies above the "knee" the distance between the tracks is not sensitive to the energy of the primary, thus not enough to estimate a lower energy threshold for a cluster. To this extent a global analisys, involving also track multiplicity and disk thickness, is being extensively studied through a set of Corsika EAS samples.

On the other side, the observation of multitracks events on single telescopes is alteady a good marker for the identification of PeV EAS.

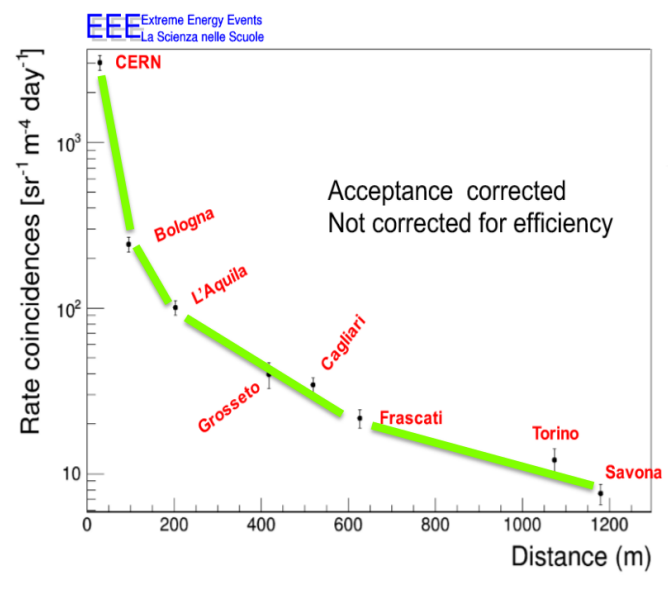

(a)

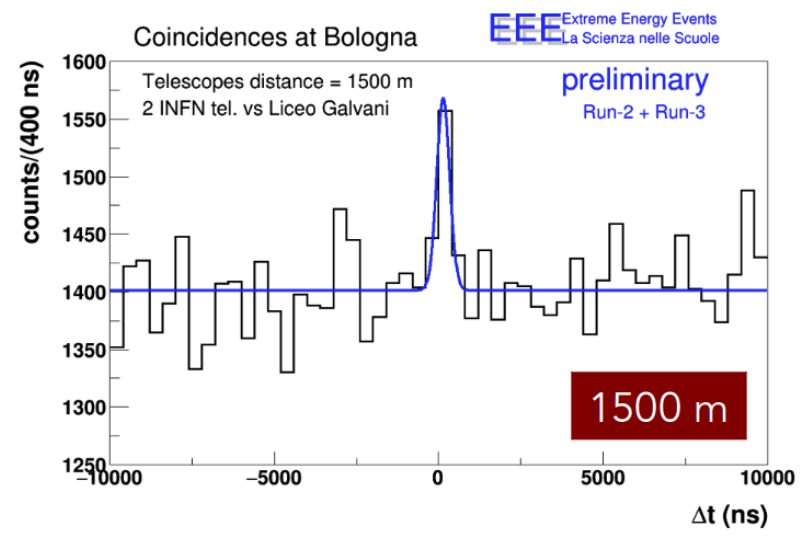

(b)

Figure 5: a). The coincidence rate between pairs of telescopes in the EEE network vs the relative distances. The results is corrected by the acceptance but is not taking into account the various efficiencies of the stations. b). Coincidence peak for a pair of telescopes in Bologna, at a relative distance of $1.5 \mathrm{~km}$.

\subsection{First studies on Long Distance Correlations}

The observation of cosmic rays time correlations between detectors at distances much larger than the the footprint of the highest energy EAS (a few $\mathrm{km}$ ) is suggested by several hypothesis, which in principle could justify the existence of such events. One of the candidate mechanisms was proposed by Gerasimova and Zatsepin [13]. In their picture, a heavy primary nucleus is photodisintegrated by interacting with solar photons. The two particles could then propagate to the 
Earth atmosphere producing two time-correlated showers, reaching the ground at very large distances.The result of numerical calculations estimate that the distances between the two showers may fall in a range from a few $\mathrm{km}$ to several hundreds $\mathrm{km}$, at extremely low rates, of the order of few events per year. Alternative models propose single sources emitting two correlated particles or massive dark matter decay as possible mechanisms for such events. No detailed numerical calculations are available for these processes.

The EEE network, thanks to the inhomogeneous topology, shows 10 sites where two or more telescopes are installed, the EEE clusters. This corresponds to a total amount of 45 pairs of clusters. Each clusters can identify EAS by selcting coincidences between tracks observed by the two telescopes of the same clusters. Then, the search of time correlated EAS can be performed by identifying coincidences between the observed EAS. The background of spurious EAS coincidences among clusters is low, from $2 \mathrm{y}^{-1}$ for a $1 \mathrm{~ms}$ time window down to $10^{-2} \mathrm{y}^{-1}$ for $\sim \mu$ s window. In Figure 6a the EEE clusters map is shown, while Figure 6b-upper shows the cluster distances distribution. The analysis approach consisits in studying the spurious coincidence rate as a function of the time window chosen, as shown in Figure 6b-lower. Then, in case of coincidences below $1 \mathrm{~ms}$ windows, the compatibility of such residual events with a statistical background, is performed. If a significant event is present, an increase in the number density at short distance should appear.

The next RUN IV, starting in October 2017, should double the statistics preliminarly used for this analysis, unveiling possible events or constraining the present upper limit of the phenomenon.

\section{Conclusions}

EEE is a rapidly growing observatory, in terms of both surface coverage and scientific topics addressed. The stations are tracking detectors at high time resolution. Since 2014 the observatory is regularly taking data in coordinated runs, gathering more than 48 billions of candidate tracks. First results have been published and several analysis are ongoing, covering low energy CR physics and solar survey as well as the study of EAS at the "knee", local anisotropies etc. The inhomogenuous topology of the network and the peculiar tracking and timing capabilities allow also for studying rare phenomena like EAS correlations, so far never observed.

The EEE Project has also an educational and outreach aim, involving high school students and teachers in constructing and taking care of the stations, while also accessing the data for educational purposes.

\section{References}

[1] Centro Studi e Ricerche "E. Fermi" web site: http://www.centrofermi.it/eee.

[2] M. Abbrescia et al., Nucl. Instrum. Meth. A 588 (2008) 211.

[3] A. Zichichi, Progetto "La Scienza nelle Scuole" - EEE: Extreme Energy Events (Società Italiana di Fisica, Bologna, 2004) 2nd edition (2005), 3rd edition (2012).

[4] ALICE Collaboration, Addendum to TOF Technical Design Report, CERN/LHCC, 2002-016.

[5] A. Akindinov et al., Nucl. Instrum. Methods Phys. Res. A 661, S98 (2012).

[6] M. Abbrescia, et al., (EEE collaboration), Nuclear Instruments and Methods in Physics Research Section A 593 (2008) 263. 


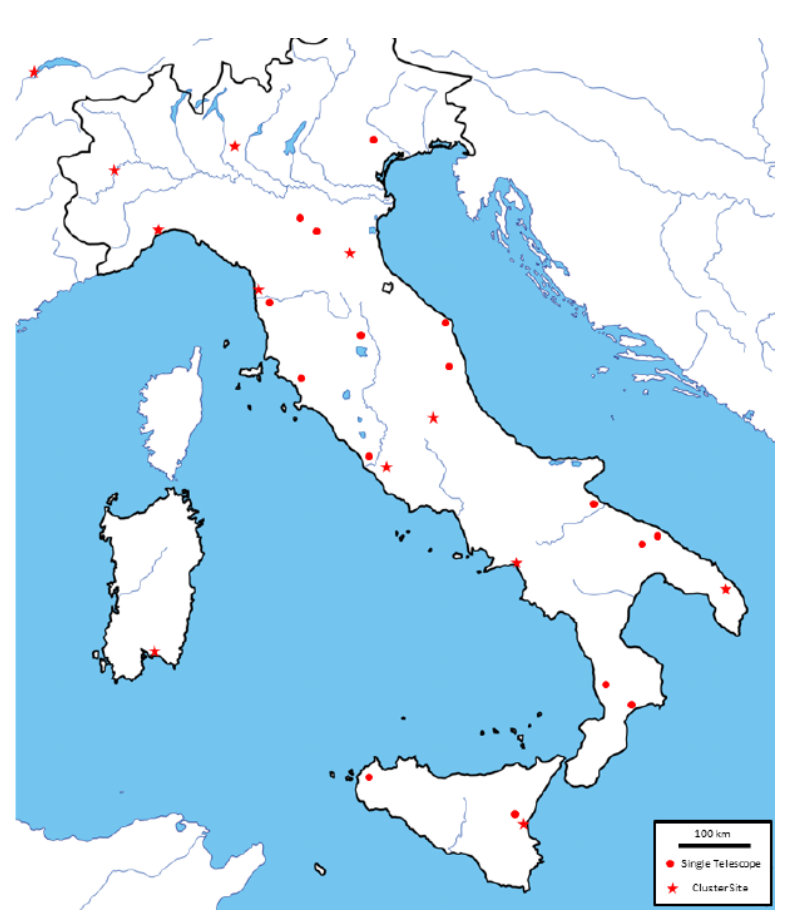

(a)
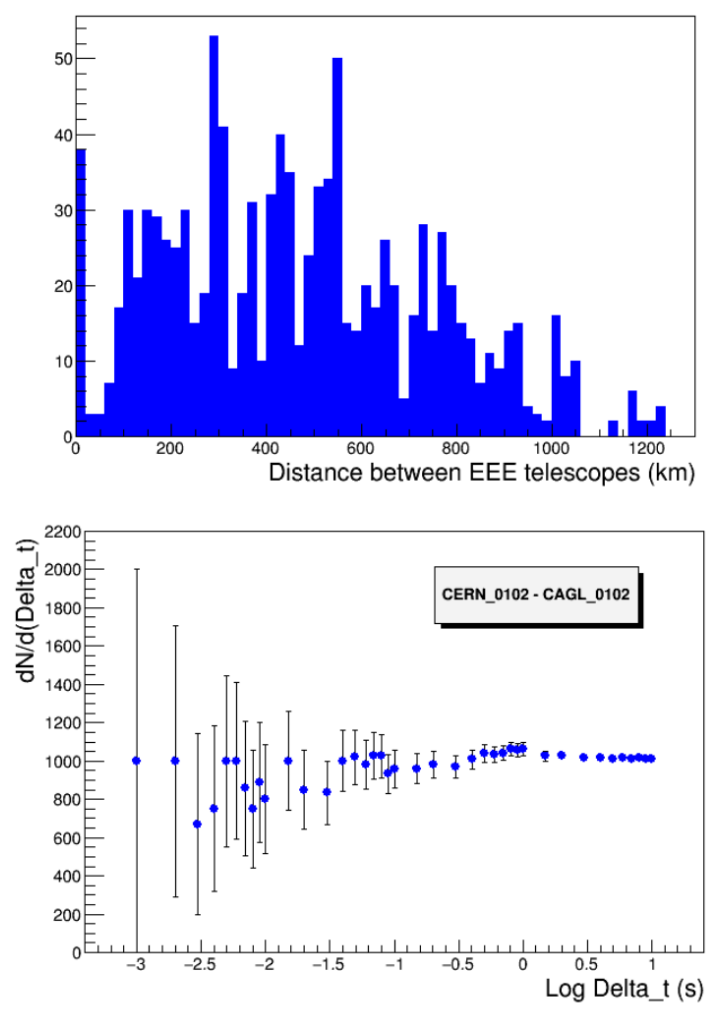

(b)

Figure 6: a). Distribution of clusters (stars) and single stations (points). b)-upper. Distribution of realtive distances among all the EEE telescopes. b)-lower. Time-density of EAS coincidences between the CERN and Cagliari EEE sites. If any non-spurious event appears, an increase in density at short $\Delta \mathrm{t}$ is expected.

[7] M. Abbrescia, et al., (EEE collaboration), Nuclear Instruments and Methods in Physics Research Section A 588 (2008).

[8]

[9] F. Anghinolfi, P. Jarron, A. N. Martemyanov, E. Usenko, H. Wenninger, M. C. S. Williams and A. Zichichi et al., Nucl. Instrum. Meth. A 533 (2004) 183.

[10] Badruddin, Nuovo Cimento C 23, 217 (2000).

[11] J. A. Lockwood and W. R. Webber, J.Geophys.Res.Space Phys. 89, A1 (1984) 17.

[12] A.H. Compton, I.A. Getting, Phys. Rev. 47, 817 (1935)

[13] N.M.Gerasimova and G.T.Zatsepin, Sov. Phys. JETP 11(1960)899. 